\title{
The influence of a gluten-free diet on health-related quality of life in individuals with celiac disease
}

\author{
Fahdah F. Al-sunaid ${ }^{1}$, Maha M. Al-homidi ${ }^{1}$, Rawan M. Al-qahtani ${ }^{1}$, Reema A. Al-ashwal ${ }^{1}$, Ghada A. Mudhish ${ }^{1}$, \\ Mahitab A. Hanbazaza ${ }^{2}$ and Abeer S. Al-zaben ${ }^{1^{*}}$
}

\begin{abstract}
Background: Adherence to a gluten-free diet (GFD) and food insecurity (FI) may influence health-related quality of life $(\mathrm{HRQOL})$ in individuals with celiac disease (CD). This study aimed to investigate the association between adherence to a GFD, $\mathrm{Fl}$, and $\mathrm{HRQOL}$ in individuals with $\mathrm{CD}$.

Methods: This cross-sectional study included 97 adults (mean age: $34 \pm 9$ years) diagnosed with CD. The participants were on a GFD for more than 6 months. Sociodemographic characteristics and medical history were assessed. Adherence to a GFD, FI, and HRQOL were assessed using validated questionnaires.

Results: Most participants (73\%) adhered to a GFD, and 62\% were experiencing FI. Individuals with CD faced difficulty in accessing GF foods due to the high cost (90\%) and limited availability (79\%). The mean overall HRQOL score was 60 . Scores on the physical and mental health domains were 69 and 47, respectively. Adherence to a GFD was significantly associated with $\mathrm{FI}(P=0.02)$, while there was no association between adherence to a GFD and HRQOL measures $(P>0.05)$. Participants facing $\mathrm{Fl}$ had lower scores in emotional well-being and mental health domains, and overall HRQOL $(P<0.05)$.

Conclusions: The findings of the present study demonstrate that Fl influences adherence to a GFD, and that FI is associated with HRQOL in terms of both emotional well-being and mental health.
\end{abstract}

Keywords: Celiac disease, Health-related quality of life, Gluten-free diet, Food insecurity

\section{Background}

Celiac disease $(C D)$ is an autoimmune disease that affects the small intestine, whereby gluten ingestion leads to the destruction of enterocytic villi in affected patients [1]. The primary genes involved in $C D$ development are HLA DQ2 and HLA DQ8 $[1,2]$. CD occurs in 1 to 2 individuals per 100 worldwide; however, systemic reviews reported that the prevalence of $\mathrm{CD}$ in Saudi Arabia is

*Correspondence: abalzaben@gmail.com

${ }^{1}$ Clinical Nutrition Program, Department of Health Sciences, College of Health and Rehabilitation Sciences, Princess Nourah Bint Abdulrahman University (PNU), PO Box 84428, Riyadh, Kingdom of Saudi Arabia Full list of author information is available at the end of the article higher (approximately 3\%) [3, 4]. Individuals with CD may experience a broad variety of gastrointestinal and malabsorption symptoms (classical CD) or extraintestinal symptoms (non-classical CD) [5]. The only available treatment for $\mathrm{CD}$ is lifelong adherence to a gluten-free diet (GFD) [6]. Adherence to a GFD refers to strict elimination of products that contain gluten such as wheat, barley, rye, and foods that are derivatives of grains (e.g., semolina, durum, spelt, triticale, and malt) [6, 7]. In previous studies, the rate of adherence to a GFD varied from 44 to $90 \%$ in patients with CD $[8,9]$. These studies have highlighted various factors that influence adherence to a GFD, such as avoidance to travel and accepting 
invitations due to strict requirements of a GFD, limited availability of gluten-free products, duration of disease, age at diagnosis, and region of residence [8-12]. The limited availability of gluten-free products may also influence food security among patients with $\mathrm{CD}$ attempting to adhere to a GFD [8].

Food insecurity (FI) is a social and economic condition that leads to restricted access to nutritious food [13]. The concept of FI includes food affordability, availability, and the acceptability of foods within one's culture [14]. Food security is achieved when all individuals have financial, physical, and social access to adequate food for maintaining nutritional requirements and a healthy life at any given time [15]. Patients with CD may face difficulty due to the higher cost and limited availability of gluten-free foods, which may in turn affect their emotional state and health-related quality of life (HRQOL) [16-19]. Decreased physical health due to gastrointestinal symptoms may also impact HRQOL in patients with CD [20]. In addition, there is strong evidence that $\mathrm{CD}$ is associated with depression, anxiety, social pressure, and difficulties in daily social relations due to the nature of the disease and/or adherence to a GFD [19]. Finally, economic status may also influence social and emotional states among patients with $\mathrm{CD}$, leading to lower HRQOL in these domains [21].

Although $\mathrm{CD}$ and its potential complications may negatively affect HRQOL, information regarding the association between adherence to a GFD, FI, and HRQOL is limited in Saudi Arabia. Therefore, this study aimed to assess the association between adherence to a GFD, FI, and HRQOL in a cohort of individuals with CD living in Saudi Arabia.

\section{Methods}

\section{Study design and participants}

This pilot cross-sectional study included 97 adult individuals with CD living in Saudi Arabia. Participants were recruited from the Saudi Celiac Association and an online support group. This approach was similar to that of the other study conducted in the UK [22]. The Saudi Celiac Association distributed the survey to members who have $C D$ and participants who were interested in this study had contacted us. After explaining the study to the participants via phone, the participants agreed to enroll in this study. The link of the consent form and the survey was distributed via What's App messages. Inclusion criteria were as follows: age 18 to 65 years, residence in Saudi Arabia, and diagnosis of CD (biopsy and/or serology) more than 6 months prior to study enrolment to guarantee adequate GFD knowledge. Participants with any other condition that can affect HRQOL (e.g., multiple food allergies, diabetes mellitus type 1 , inflammatory bowel disease, hepatitis $\mathrm{C}$, multiple sclerosis, coronary artery disease, end-stage renal disease, stroke, kidney transplant, and hemodialysis treatment) were excluded. All subjects gave their informed consent for inclusion before they participated in the study. The study was conducted in accordance with the Declaration of Helsinki, and the protocol was approved by the Ethics Committee of Princess Nourah bint Abdulrahman University (H-01-R-059), IRB log number (20-0011).

\section{Sociodemographic characteristics and medical history}

Data related to sociodemographic factors were collected including age, gender, nationality, education level, marital status, area of residence, and monthly family income. Anthropometric information including weight and height was self-reported. Medical history was assessed based on the family history of $\mathrm{CD}$, age at $\mathrm{CD}$ diagnosis, comorbid diseases such as diabetes mellitus, food allergies, or food intolerance, and whether patients were symptomatic (abdominal bloating, diarrhea, headache, and flatulence) or asymptomatic [22].

\section{Adherence to a GFD}

Adherence to a GFD was assessed using the validated Biagi questionnaire, which is a simple survey consisting of four questions [23]. The questionnaire was translated to Arabic and back translated. After translation, the questionnaire was sent to the participants in Arabic. Total scores in this questionnaire range from 0 to 5 . Scores of $0-1$ indicate that the patient does not strictly adhere to a GFD, while a score of 2 indicates that the patient follows a GFD but requires further education based on a question asking the participants if they do read and check food labels on packaged food. Scores of 3-5 reflect strict adherence to a GFD [23].

\section{Fl status}

FI status was assessed using the Arabic Version of the Food Insecurity Experience Scale Survey Module (FIESSM) [21]. The FIES-SM has been validated in Middle Eastern countries [24-26]. The FIES-SM contains eight items related to food consumption over the last 12 months. The response to these questions was yes or no (1 vs. 0$)$. Final scores range from 0 to 8 based on the affirmative response. Scores $0-1$ indicate food security, 2-3 indicate mild FI (worrying about the ability to obtain food), 4-6 moderate FI, and 7-8 indicate severe FI (hunger) [27]. We included two additional questions adapted from previous studies $[16,28]$. The first question assessed the accessibility of gluten-free processed foods (glutenfree: bread, pasta, breakfast cereal, flour, and snacks) in grocery stores in Saudi Arabia. Three possible answers were included: very accessible, somewhat accessible, and 
not accessible. The second question assessed the seven challenges individuals with CD face to afford a GFD: high price, distance to grocery stores, physical disabilities, not available, limited variety of items, low quality of GFD, or/ and not provided in the hospitals.

\section{Assessment of HRQOL}

HRQOL was assessed using the 36-Item Short Form Survey (SF-36), which includes 36 questions across eight scales: physical functioning, role limitations due to physical health, pain, general health, role limitations due to emotional problems, energy/fatigue, emotional wellbeing, and social functioning. The items were further clustered into two summary domains (physical health and mental health), and the overall HRQOL was calculated based on the average of physical and mental health domains. All items were scored from 0 to 100 , with higher scores indicating better HRQOL [29-31]. SF-36 was validated and used previously in Saudi population [32].

\section{Statistical analysis}

Data were analyzed using the Statistical Package for the Social Sciences (SPSS) version 25 (2017). $P$ values $<0.05$ were considered statistically significant. Normally distributed variables are presented as mean $( \pm S D)$, while skewed variables are presented as median and interquartile range (IQR). Due to the small sample size of some groups when assessing education levels and areas of residence, patients were classified into two groups when assessing the association between these factors and adherence to GFD, FI, and HRQOL. For analyses of education levels, we compared patients who had obtained at least a bachelor's degree with those who had not. For residential analyses, we compared patients residing in central regions with those residing in non-central regions (i.e., south, east, west, and north regions). Mann-Whitney U test was used to compare non-normally distributed variables, while the independent $\mathrm{t}$-test was used to compare normally distributed variables. Chi-square test was used to compare categorical variables. Correlation test was performed to assess the association between the continuous sociodemographic, anthropometric, and medical history variables (age, body mass index (BMI), and duration of the disease), and HRQOL.

\section{Results}

\section{Sociodemographic characteristics and medical history}

One hundred and fifteen participants who were interested in this study were enrolled. One participant who was $<18$ years old and 17 participants in whom CD was diagnosed within 6 months prior to enrollment were excluded. Ninety-seven participants met the inclusion criteria and were included in the study. Table 1 displays the sociodemographic characteristics of the included participants. Most participants were female. Underweight status was noted in $13 \%$ of participants, while overweight and obese status was noted in $28 \%$ and $15 \%$ of participants, respectively. Most participants were of Saudi descent, had at least a bachelor's degree, were married, lived in the central region of the country, and had a monthly income less than 5,000 Saudi Riyals (SAR).

Forty-two participants (43\%) had a family history of CD. Symptoms related to CD such as diarrhea, headache, and flatulence appeared in most participants $(n=63$;

Table 1 Sociodemographic characteristics of participants with celiac disease living in Saudi Arabia $(n=97)$

\begin{tabular}{|c|c|}
\hline Variables & $\begin{array}{l}\text { N number (\%) or } \\
\text { Mean } \pm \text { Standard } \\
\text { division }\end{array}$ \\
\hline Age (years) & $34 \pm 9$ \\
\hline Weight (kg) & $60 \pm 15$ \\
\hline Height (cm) & $158 \pm 8$ \\
\hline BMI $\left(\mathrm{kg} / \mathrm{m}^{2}\right)$ & $24.2 \pm 5.3$ \\
\hline \multicolumn{2}{|l|}{ Gender } \\
\hline Male & $11(11 \%)$ \\
\hline Female & $86(89 \%)$ \\
\hline \multicolumn{2}{|l|}{ Nationality } \\
\hline Saudi & $92(95 \%)$ \\
\hline Non-Saudi & $5(5 \%)$ \\
\hline \multicolumn{2}{|l|}{ Education Level } \\
\hline Intermediate school & $7(7 \%)$ \\
\hline High school graduate & $22(23 \%)$ \\
\hline Bachelor's degree & $53(55 \%)$ \\
\hline Diploma & $10(10 \%)$ \\
\hline Higher education (Master and PhD) & $5(5 \%)$ \\
\hline \multicolumn{2}{|l|}{ Marital status } \\
\hline Single & $34(35 \%)$ \\
\hline Married & $60(62 \%)$ \\
\hline Divorced & $3(3 \%)$ \\
\hline Widowed & $0(0 \%)$ \\
\hline \multicolumn{2}{|l|}{ Regions of Residence } \\
\hline Central region & $70(72 \%)$ \\
\hline South region & $9(9 \%)$ \\
\hline East region & $6(6 \%)$ \\
\hline West region & $7(7 \%)$ \\
\hline North region & $5(5 \%)$ \\
\hline \multicolumn{2}{|l|}{ Monthly family income } \\
\hline Less than 5,000 SAR/month & $53(55 \%)$ \\
\hline 5,000 to $10,000 \mathrm{SAR} /$ month & $21(21 \%)$ \\
\hline More than $10,000 \mathrm{SAR} /$ month & $23(24 \%)$ \\
\hline
\end{tabular}

Data are presented as mean \pm SD or number of participants (\%) BMI, body mass index; SAR, Saudi Riyals; SD, standard deviation 
$65 \%)$, while only $35 \%$ were asymptomatic $(n=34)$. While the majority of the participants $(n=73,75 \%)$ had $\mathrm{CD}$ alone with no other comorbidities, 24 participants had CD with other diseases (lactose intolerance or one food allergy (e.g. egg). The mean age at diagnosis was $28 \pm 11$ years (range: $5-51$ years). The mean duration of following GFD was $6 \pm 5$ years (range: $1-37$ years).

\section{Adherence to a GFD}

Approximately $27 \%(n=26)$ of participants were not adhering to a GFD. Among the 71 participants (73\%) adhering to a GFD, none exhibited low knowledge. Individuals adhering to a GFD were significantly older (36 \pm 9 years) than those not adhering to such a diet ( $29 \pm 7$ years) $(P=0.001)$. No significant association was observed between adherence to a GFD and any other sociodemographic factors (weight, height, BMI, educational level, marital status, region of residence, and income) $(P>0.05)$. Moreover, medical history (family history of $\mathrm{CD}$, age at $\mathrm{CD}$ diagnosis, related comorbidities, and symptomatic status) was not significantly associated with adherence to a GFD $(P>0.05)$.

\section{FI status}

Figure 1 represents the food insecurity status in individuals with CD assessed using the Food Insecurity Experience Scale Survey Module. Only 38\% of participants $(n=37)$ reported food security. Thus, $62 \%$ of participants $(n=60)$ faced FI in the present study. Most participants with FI $(n=40,76 \%)$ had family incomes below 5,000

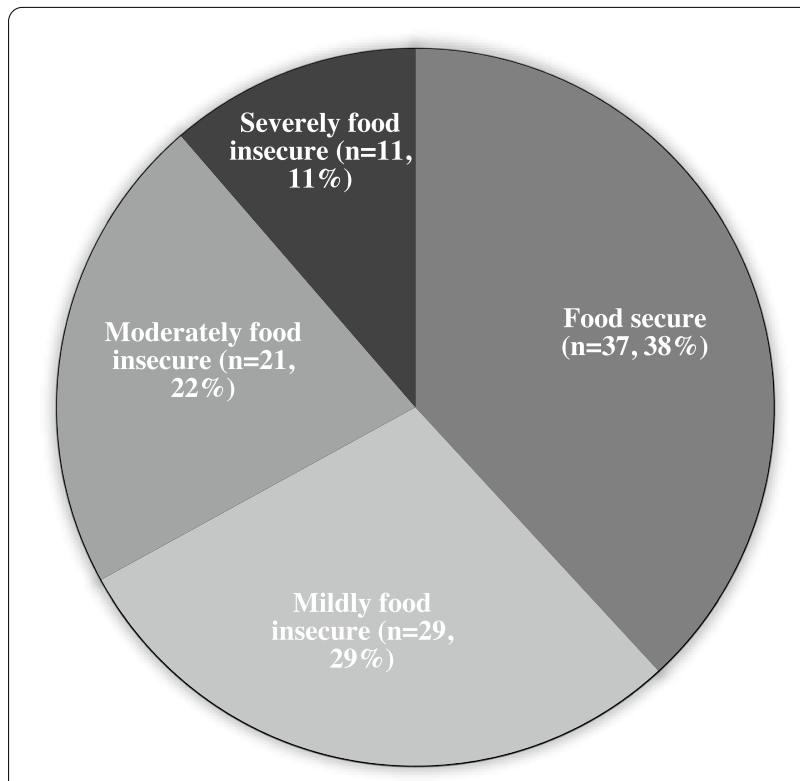

Fig. 1 Fl status in individuals with CD assessed using the Food Insecurity Experience Scale Survey Module $(n=97)$
SAR/month, while most with food security $(n=14,61 \%)$ had incomes over $10,000 \mathrm{SAR} /$ month $(P=0.007)$. Individuals living in the central region of Saudi Arabia were significantly more food secure $(n=31,84 \%)$ than patients living in non-central regions $(n=6,16 \%)(P=0.045)$. No significant association was observed between FI and sociodemographic characteristics (age, weight, height, BMI, nationality, educational level, or marital status) or medical history (family history of CD, age at CD diagnosis, presence of related comorbidities, symptomatic status) $(P>0.05)$.

Figure 2 shows the accessibility of gluten-free processed foods among participants with CD. Gluten-free pasta $(n=46,47 \%)$ and gluten-free breakfast cereal $(n=43,44 \%)$ were the two most accessible foods from grocery stores in Saudi Arabia. Figure 3 illustrates the challenges that participants faced in accessing glutenfree food. Gluten-free foods were not available in the supermarket for more than half of participants $(n=53$, $55 \%)$. Additional challenges to access gluten-free foods included travel, lack of Arabic breads, and limited glutenfree options at restaurants.

\section{HRQOL}

Table 2 displays HRQOL findings in participants with CD. Age $(r=-0.256, P=0.012)$ and BMI $(r=-0.221$, $P=0.035)$ exhibited significant weak inverse correlations with physical functioning scores. Women scored significantly lower than men on physical functioning (women: median, 75 [IQR, 50-95], men: median, 100 [IQR, 90-100], $P=0.001$ ) and emotional well-being scales (women: $54 \pm 20$, men: $70 \pm 18, P=0.014$ ). Participants who had obtained at least a bachelor's degree scored significantly higher on the emotional well-being scale than individuals who had not attained a bachelor's degree (bachelor's degree: $59 \pm 20$, without a bachelor's degree: $51 \pm 19, P=0.041)$. Participants living in the central region of the country had significantly higher HRQOL scores than those living in non-central regions on the following domains: role limitations due to physical health problems (central: median, 100 [IQR, 25-100], non-central: median, 25 [IQR, $0-100], P=0.011)$, energy/fatigue (central: median, 50 [IQR, 39-60], non-central: median, 40 [IQR, 30-50], $P=0.016$ ), social functioning (central: median, 63 [IQR, 50-88], non-central: median, 50 [IQR, 38-75], $P=0.039$ ), physical health (central: median, 73 [IQR, 50-88], non-central: median, 56 [IQR, 44-78], $P=0.033$ ), and mental health (central: median, 50 [IQR, 39-74], non-central: median, 40 [IQR, 26-69], $P=0.037$ ). The income was categorized to three categories: $<5,000$ $\mathrm{SAR} /$ month, 5,000 to $10,000 \mathrm{SAR} /$ month, and $>10,000$ SAR/month. Participants with a higher income $(>10,000$ 


\section{Accessiblity of gluten-free foods}

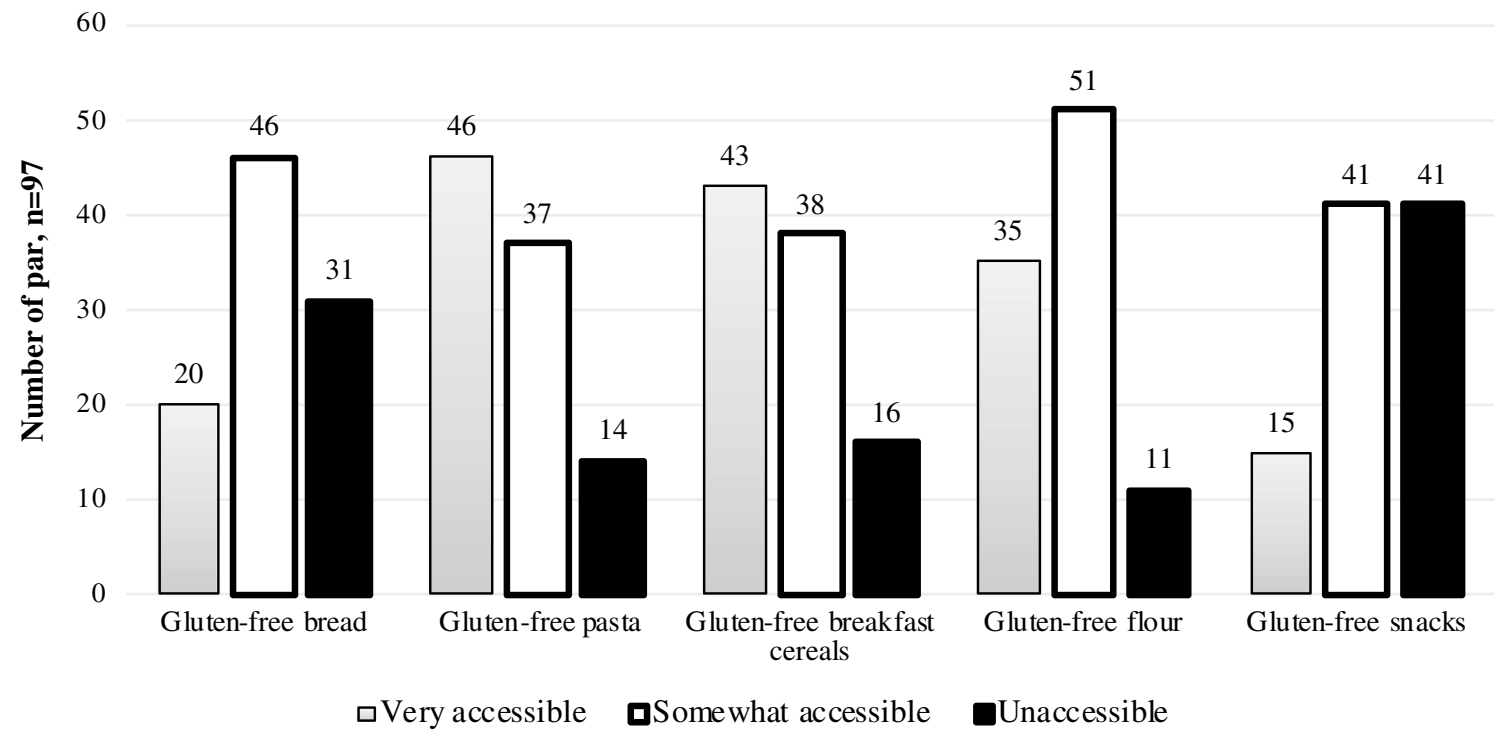

Fig. 2 The accessibility of gluten-free processed foods among participants with $C D(n=97)$. X-axis represents the gluten-free processed foods and Y-axis represents the number of participants

\section{Affordability challenges of gluten-free foods}

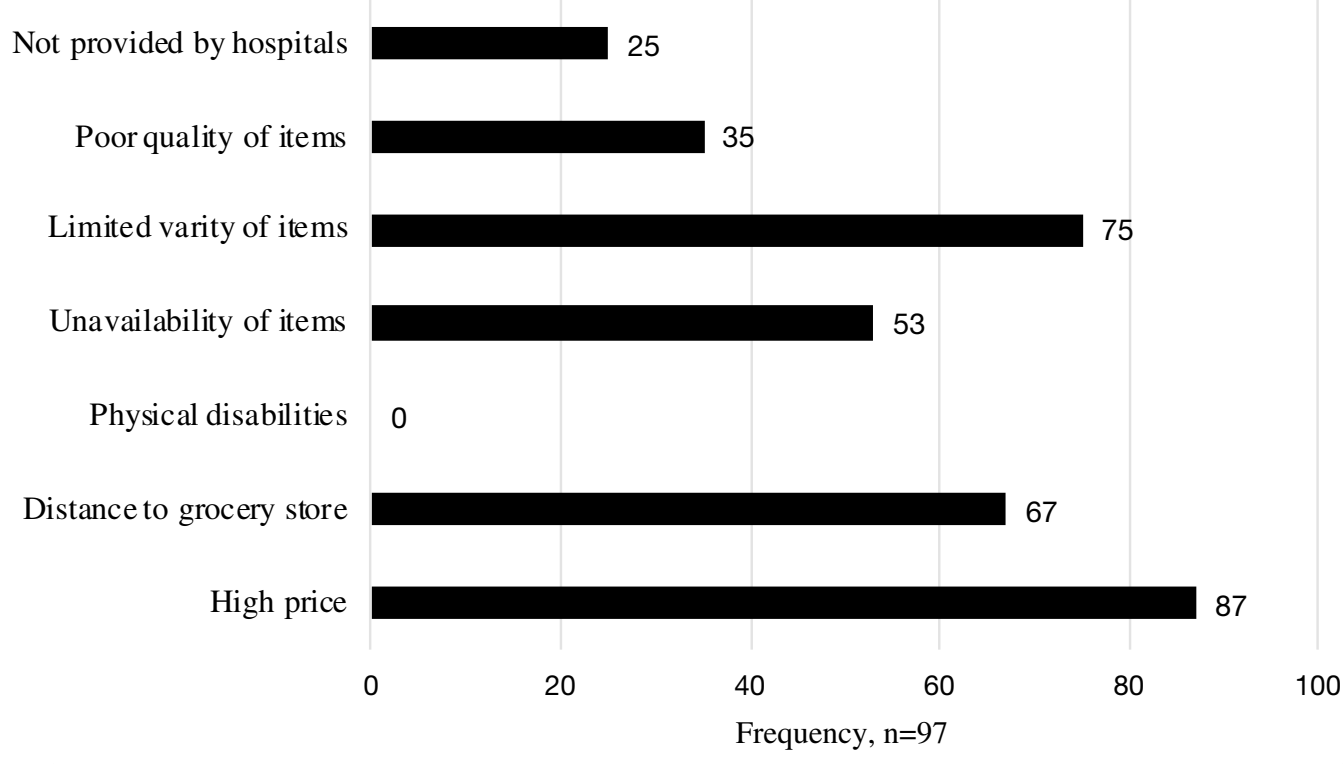

Fig. 3 Factors influencing affordability of gluten-free foods in participants with celiac disease $(n=97)$. X-axis represents the number of participants and $\mathrm{Y}$-axis represents the factors influencing affordability of gluten-free foods

SAR/month) scored significantly higher on physical functioning (median, 95 [IQR, 85-100], median, 65 [IQR, 43-75], median, 80 [IQR, 50-100], $P=0.000)$, energy/ fatigue (median, 60 [IQR, 50-70], median, 45 [IQR,
30-50], median, 45 [IQR, 35-53], $P=0.000$ ), emotional well-being $(67 \pm 15,55 \pm 18,52 \pm 21, P=0.009)$, pain (median, 90 [IQR, 68-100], median, 58 [IQR, 45-79], median, 78 [IQR, 55-89], $P=0.04)$, physical health 
Table 2 Health-related quality of life based on Short Form 36 findings in participants with celiac disease living in Saudi Arabia ( $\mathrm{n}=97$ )

\begin{tabular}{|c|c|c|c|c|c|c|}
\hline SF-36 & $\begin{array}{l}\text { Patients with CD } \\
(n=97)\end{array}$ & $\begin{array}{l}\text { Adherence to } \\
\text { GFD }(n=71)\end{array}$ & $\begin{array}{l}\text { Non-adherence } \\
\text { to GFD }(n=26)\end{array}$ & $P$ value $^{a}$ & $\begin{array}{l}\text { Food secure } \\
(n=37)\end{array}$ & $\begin{array}{l}\text { Food insecure } \\
(n=60)\end{array}$ \\
\hline Physical functioning ${ }^{b}$ & $80(58-100)$ & $80(50-100)$ & $8765-100)$ & 0.435 & $85(65-100)$ & $75(51-95)$ \\
\hline Role limitation due to physical health problems ${ }^{\mathrm{b}}$ & $75(13-100)$ & $75(25-100)$ & $75(0-100)$ & 0.851 & $100(25-100)$ & $63(0-100)$ \\
\hline Role limitation due to emotional problems ${ }^{\mathrm{b}}$ & $33(0-100)$ & $67(0-100)$ & $0(0-100)$ & 0.083 & $67(0-100)$ & $0) 0-100)$ \\
\hline Energy/fatigue $^{b}$ & $50(35-60)$ & $50(35-60)$ & $45(40-50)$ & 0.344 & $55(38-60)$ & $45(35-54)$ \\
\hline Emotional well-being ${ }^{c}$ & $56 \pm 20$ & $56 \pm 21$ & $55 \pm 17$ & 0.734 & $62 \pm 20$ & $52 \pm 19$ \\
\hline Social functioning ${ }^{b}$ & $63(38-88)$ & $63(38-88)$ & $63(50-88)$ & 0.557 & $75(50-88)$ & $63(38-75)$ \\
\hline Pain ${ }^{b}$ & $78(55-90)$ & $78(58-90)$ & $68(45-83)$ & 0.209 & $68(58-90)$ & $78(48-90)$ \\
\hline General health ${ }^{c}$ & $60 \pm 18$ & $62 \pm 19$ & $55 \pm 16$ & 0.102 & $64 \pm 20$ & $57 \pm 16$ \\
\hline Physical health domain ${ }^{b, d}$ & $69(48-85)$ & $69(50-85)$ & $68(46-85)$ & 0.557 & $73(60-86)$ & $66(48-85)$ \\
\hline Mental health domain ${ }^{b, e}$ & $47(35-73)$ & $52(35-74)$ & $45(35-67)$ & 0.404 & $68(40-79)$ & $45(35-64)$ \\
\hline Overall HRQOL score ${ }^{c, f}$ & $60 \pm 21$ & $61 \pm 22$ & $57 \pm 19$ & 0.447 & $65 \pm 21$ & $56 \pm 20$ \\
\hline
\end{tabular}

$\mathrm{CD}$, celiac disease; GFD, gluten-free diet HRQOL, health-related quality of life; $n$, number of participants; $S F-36$, 36-item short form survey

a $P$ values $<0.050$ are considered statically significant

${ }^{\mathrm{b}}$ Variables demonstrating skewed distributions are presented as median (interquartile range)

' Normally distributed variables are presented as mean \pm standard deviation

${ }^{\mathrm{d}}$ The average of physical functioning, role limitations due to physical health problems, pain, and general health

${ }^{\mathrm{e}}$ The average of role limitations due to emotional problems, energy/fatigue, emotional well-being, and social functioning

${ }^{\mathrm{f}}$ The average of physical health domain and mental health domain

(median, 85 [IQR, 66-89], median, 53 [IQR, 44-74], median, 78 [IQR, 55-89], $P=0.003)$, and mental health domains (median, 72 [IQR, 44-85], median, 45 [IQR, 30-68], median, 45 [IQR, 32-67], $P=0.015$ ) than those with lower incomes of 5,000-10,000 SAR or $<5,000$ SAR per month, respectively. Marital status and medical history were not significantly associated with HRQOL $(P>0.05)$.

\section{Association between $\mathrm{FI}$, adherence to a GFD, and HRQOL}

Table 2 shows the relationship between adherence to a GFD and HRQOL, and between FI and HRQOL. Adherence to a GFD was not significantly associated with any HRQOL scale or domain $(P>0.05)$. Participants facing FI had significantly lower emotional well-being, mental health, and overall HRQOL scores than those with food security $(P<0.05)$. There was a significant association between adherence to a GFD and FI $(P=0.02)$. Most participants not adhering to a GFD were FI $(n=21,81 \%)$, while almost half of the participants adhering to a GFD were food secure $(n=32,45 \%)$ (Table 3$)$.

\section{Discussion}

This study aimed to assess the influence of adherence to a GFD, FI, and sociodemographic characteristics on HRQOL in patients with CD. Our findings indicated that most participants (73\%) were adherent to a GFD, although $62 \%$ were experiencing mild to severe FI. Furthermore, FI and age significantly influenced the adherence to a GFD. Participants with low monthly income
Table 3 Association between food insecurity and adherence to a gluten-free diet

\begin{tabular}{lclll}
\hline & $\begin{array}{l}\text { Non-adherence } \\
\boldsymbol{n}(\%)\end{array}$ & $\begin{array}{l}\text { Adherence } \\
\boldsymbol{n}(\%)\end{array}$ & $\begin{array}{l}\text { Total } \\
\boldsymbol{n}(\%)\end{array}$ & P value \\
\hline Food Secure & $5(19)$ & $32(45)$ & $37(38)$ & $0.020^{*}$ \\
Food Insecure & $21(81)$ & $39(55)$ & $60(62)$ & \\
Total & $26(27)$ & $71(73)$ & $97(100)$ & \\
\hline
\end{tabular}

$\mathrm{n}$, number of participants

${ }^{*} P$ values $<0.05$ are considered statically significant

and those living in non-central regions of Saudi Arabia were more FI and had a poorer HRQOL. FI was significantly associated with both poor adherence to a GFD and poor overall HRQOL. Although adherence to a GFD was not directly associated with HRQOL, FI was significantly associated with HRQOL.

In this study, most of the participants reported FI. Unfortunately, there is no national information about FI in Saudi Arabia; therefore, comparing the result of the present study to the national prevalence of FI was not possible. To our knowledge, few studies have assessed the impact of FI among individuals with $\mathrm{CD}$. In one international study, FI was noted in $42 \%$ of 15,819 individuals residing in the Middle East and North Africa-a rate lower than that noted in the presented study $(62 \%)$ [14]. Previous studies have also indicated that food availability, affordability, and acceptability can influence FI [33]. In our study, participants reported that gluten-free snacks and bread were the two most inaccessible foods in 
grocery stores in Saudi Arabia. In addition, participants reported several challenges in accessing gluten-free foods in Saudi Arabia, such as high cost and limited availability. These findings are similar to those of a prior study conducted in the UK [16]. Although some studies have indicated that adherence to a GFD may influence FI due to the high cost and limited availability of glute-free foods $[14,16]$, it is difficult to compare our findings given the dearth of research related to FI among individuals with $\mathrm{CD}$ in different countries.

In accordance with previous findings among individuals with CD in the US, Italy, and Brazil [23, 34, 35], most participants in our study adhered to a GFD. Similar to our finding, one previous study noted that $72 \%$ of Saudi children with CD adhere to a GFD [36]. However, the adherence to GFD in adults with CD ranged from 30 to $90 \%$ in previous studies $[8,9,37-40]$. Such studies have highlighted the potential influence of sociodemographic factors (age, area of residence, age at diagnosis, presence of symptoms during gluten exposure, GFD-related comorbidities, and participation in support groups) on GFD adherence [10-12, 40]. However, in the present study, age was the only sociodemographic factor associated with adherence to a GFD.

In current study, although adherence to a GFD was high, FI was observed in the majority of the participants, and most participants who did not adhere to a GFD showed FI. These findings suggest that FI is highly dependent on adherence to a GFD. Several studies have documented the influence of FI on adherence to a GFD using self-report questionnaires (e.g., Celiac Dietary Adherence Test (CDAT)) and observational methods [41-43]. Other studies have reported the association between FI and adherence to Mediterranean and DASH diets $[13,44,45]$. Low socioeconomic status may also be associated with FI, and therefore poor adherence to the Mediterranean diet [13]. Our findings also indicate that lower income levels may be associated with relatively poorer adherence to a GFD. Several studies have reported that HRQOL is poor among patients with CD [20, 22, 46, 47]. Several factors have been associated with lower HRQOL scores in these patients, including lack of adherence to a GFD, delay in CD diagnosis, and lower education levels $[20,46,48]$. Unsurprisingly, age exhibits a significant negative correlation with physical functioning, which may reflect physical deterioration due to decreased muscle mass and strength [49]. In accordance with previous findings [50], women in our study exhibited lower scores on physical functioning and emotional well-being scales than men. However, medical history (presence of symptoms and age at $\mathrm{CD}$ diagnosis) was not significantly associated with HRQOL. This may be because most participants adhered to a GFD. Although, the present study did not find any strong association between BMI and HRQOL possibly due to the small sample size, increased BMI was negatively correlated with physical functioning, as reported in previous studies [50,51]. Accumulating evidence indicates that there is an association between $\mathrm{CD}$ and non-alcoholic fatty liver disease [52], possibly due to the poor nutritional quality of GFD (high in saturated fat, calories, and simple sugars) [53].

In contrast to the current findings, several studies have reported that patients with $C D$ who adhere to a GFD exhibit better HRQOL scores than those who do not [20, $21,48]$. Although this may be because most participants adhered to a GFD, the finding may also be explained by differences in the methods used to assess GFD adherence among studies. Alternatively, the low availability and accessibility of GFD may lead to stress responses such as anxiety and depression, which may explain the association between adherence to a GFD and reduced HRQOL, especially in the emotional health domain. Indeed, previous studies have noted that increased concern related to one's ability to maintain a food supply can lead to anxiety and depression [14].

To the best of our knowledge, this study is the first to assess the association between FI, adherence to a GFD, and HRQOL in participants with CD living in the Middle East. However, our study possesses some limitations including a small sample size, and the use of self-report questionnaires to assess adherence to a GFD. Serological tests may allow a more objective assessment of GFD adherence [54]. Furthermore, the sensitivity and the specificity of the serology tests for CD diagnosis are not $100 \%$. the present study included participants with confirming $C D$ diagnosed either through a biopsy and serology $(n=24)$ or based on serology $(n=73)$. For patients with $\mathrm{CD}$ diagnosed through serology tests, the serology tests may include deaminated gliadin, deamidated gliadin peptide, or tissue transglutaminase and the cutoff values for serology tests were not reported. Additionally, the present study did not include an adequate control (non-CD) group because it is difficult to identify a control group (non-CD) following GFD. Moreover, there are no reference values for HRQOL in the Saudi population. However, the HRQOL scores in the current study was similar to those observed in another study on Saudi Arabian patients with CD [20]. Finally, the study participants were recruited from the membership of Celiac Association in Saudi Arabia and an online support group, thus, the sociodemographic and/or disease characteristics of the study population might not be reflective of the lager population with CD living in Saudi Arabia. Nevertheless, many similar studies have recruited participants from non-profit organizations and patients support 
organizations in the UK, Canada, Finland, and Sweden $[22,46,55,56]$.

\section{Conclusions}

In conclusion, our findings indicated that while most participants adhered to a GFD, they faced varying degrees of FI. Thus, FI significantly influences the adherence to a GFD. In addition, FI was associated with lower HRQOL in terms of both emotional well-being and mental health. Future studies should assess the adherence to a GFD via serological testing to avoid the influence of over- or under-reporting. Additional studies are required to establish reference values of HRQOL for healthy adults living in Saudi Arabia, as it will allow a more effective comparison among studies.

\section{Abbreviations}

CD: Celiac disease; FI: Food insecurity; HRQOL: Health-related quality of life; GFD: Gluten-free diet; FIES-SM: Food Insecurity Experience Scale Survey Module; SAR: Saudi Riyal; CDAT: Celiac Dietary Adherence Test; SD: Standard deviation; BMI: Body mass index; IQR: Interquartile range; SF-36: 36-Item Short Form Survey.

\section{Acknowledgements}

The authors wish to thank and the Celiac Association in Saudi Arabia for their help in recruiting the participants.

\section{Authors' contributions}

F.F.A. designed research, conducted research, analyzed data, wrote paper; M.M.A. designed research, conducted research, analyzed data, wrote paper; R.M.A., R.A.A., G.A.M. designed research, conducted research, wrote paper; M.A.H. designed research, analyzed data; and A.S.A designed research, analyzed data, wrote paper. A.S.A had primary responsibility for final content. All authors read and approved the final manuscript.

\section{Funding}

This research was funded by the Deanship of Scientific Research at Princess Nourah bint Abdulrahman University through the Fast-track Research Funding Program.

\section{Availability of data and materials}

The datasets used and/or analysed during the current study available from the corresponding author on reasonable request.

\section{Declarations}

\section{Ethics approval and consent to participate}

The study was conducted in accordance with the Declaration of Helsinki, and the protocol was approved by the Ethics Committee of Princess Nourah bint Abdulrahman University (H-01-R-059), IRB log number (20-0011). All subjects gave their informed consent for inclusion before they participated in the study.

\section{Consent for publication}

Not applicable.

\section{Competing interests}

The authors declare that they have no competing interests.

\section{Author details}

${ }^{1}$ Clinical Nutrition Program, Department of Health Sciences, College of Health and Rehabilitation Sciences, Princess Nourah Bint Abdulrahman University (PNU), PO Box 84428, Riyadh, Kingdom of Saudi Arabia. ${ }^{2}$ Department of Food and Nutrition, Faculty of Human Sciences and Design, King Abdulaziz University (KAU), Jeddah, Kingdom of Saudi Arabia.

Received: 20 April 2021 Accepted: 16 August 2021

Published online: 25 August 2021

References

1. Green PH, Cellier C. Celiac disease. N Engl J Med. 2007;357:1731-43.

2. Harmon GS, Lebeck LK, Weidner N. Gluten-dependent enteropathy and atypical human leukocyte antigen alleles. Hum Pathol. 2011;42:1112-6.

3. Ludvigsson JF, Ciacci C, Green PH, Kaukinen K, Korponay-Szabo IR, Kurppa $\mathrm{K}$, et al. Outcome measures in coeliac disease trials: the Tampere recommendations. Gut. 2018;67:1410-24.

4. El-Metwally A, Toivola P, AIAhmary K, Bahkali S, AlKhathaami A, AISaqabi MK, et al. The epidemiology of celiac disease in the general population and high-risk groups in Arab Countries: a systematic review. Biomed Res Int. 2020;2020:6865917.

5. Jericho H, Assiri A, Guandalini S. Celiac disease and wheat intolerance syndrome: a critical update and reappraisal. J Pediatr Gastroenterol Nutr. 2017;64:15-21.

6. Sample D, Turner J. Improving gluten free diet adherence by youth with celiac disease. Int J Adolesc Med Health. 2019

7. Alaedini A, Green PH. Narrative review: celiac disease: understanding a complex autoimmune disorder. Ann Intern Med. 2005;142:289-98.

8. Hall NJ, Rubin G, Charnock A. Systematic review: adherence to a glutenfree diet in adult patients with coeliac disease. Aliment Pharmacol Ther. 2009:30:315-30.

9. Zarkadas M, Cranney A, Case S, Molloy M, Switzer C, Graham ID, et al. The impact of a gluten-free diet on adults with coeliac disease: results of a national survey. J Hum Nutr Diet. 2006;19:41-9.

10. Kivelä L, Hekkala S, Huhtala H, Kaukinen K, Kurppa K. Lack of long-term follow-up after paediatric-adult transition in coeliac disease is not associated with complications, ongoing symptoms or dietary adherence. United European Gastroenterol J. 2020;8:157-66.

11. Leinonen H, Kivelä L, Lähdeaho ML, Huhtala H, Kaukinen K, Kurppa K. Daily life restrictions are common and associated with health concerns and dietary challenges in adult celiac disease patients diagnosed in childhood. Nutrients. 2019;11:1718.

12. Kurppa K, Lauronen O, Collin P, Ukkola A, Laurila K, Huhtala H, et al. Factors associated with dietary adherence in celiac disease: a nationwide study. Digestion. 2012;86:309-14.

13. Gregório MJ, Rodrigues AM, Graça P, de Sousa RD, Dias SS, Branco JC, et al. Food insecurity is associated with low adherence to the mediterranean diet and adverse health conditions in Portuguese adults. Front Public Health. 2018;6:38

14. Jones AD. Food insecurity and mental health status: a global analysis of 149 countries. Am J Prev Med. 2017;53:264-73.

15. Khalifeh F, Riasatian MS, Ekramzadeh M, Honar N, Jalali M. Assessing the prevalence of food insecurity among children with celiac disease: a crosssectional study. J Food Secur. 2019;7:192-5.

16. Singh J, Whelan K. Limited availability and higher cost of gluten-free foods. J Hum Nutr Diet. 2011;24:479-86.

17. Estévez V, Ayala J, Vespa C, Araya M. The gluten-free basic food basket: a problem of availability, cost and nutritional composition. Eur J Clin Nutr. 2016;70:1215-7.

18. Hanci $O$, Jeanes YM. Are gluten-free food staples accessible to all patients with coeliac disease? Frontline Gastroenterol. 2019;10:222-8.

19. Buchanan R, Arman J. Celiac disease: depression, anxiety, social stigma, and implications for counselors. ACA Knowl Center. 2015;4:1-8.

20. Al-Qefari SN, Al-Twijri AW, Al-Adhadh AM, Al-Rashed OA, Al-Jarallah B. Health-related quality of life among patients with celiac disease in Saudi Arabia. Ann Med Health Sci Res. 2018:8:74-7.

21. Zysk W, Głąbska D, Guzek D. Social and emotional fears and worries influencing the quality of life of female celiac disease patients following a gluten-free diet. Nutrients. 2018;10:1414.

22. Violato M, Gray A. The impact of diagnosis on health-related quality of life in people with coeliac disease: a UK population-based longitudinal perspective. BMC Gastroenterol. 2019;19:68. 
23. Biagi F, Andrealli A, Bianchi PI, Marchese A, Klersy C, Corazza GR. A glutenfree diet score to evaluate dietary compliance in patients with coeliac disease. Br J Nutr. 2009;102:882-7.

24. Naja F, Hwalla N, Fossian T, Zebian D, Nasreddine L. Validity and reliability of the Arabic version of the Household Food Insecurity Access Scale in rural Lebanon. Public Health Nutr. 2015;18:251-8.

25. Food and Agriculture Organization of the United Nations. Voices of the Hungry Applying the FIES. Food and Agriculture Organization of the United Nations. 2020. http://www.fao.org/in-action/voices-of-the-hungry/using-fies/en/. Accessed 12 Oct 2020.

26. FAO. 2019. Rural transformation-key for sustainable development in the near east and North Africa. Overview of Food Security and Nutrition 2018 Cairo. http://www.fao.org/3/ca3817en/CA3817EN.pdf. Accessed 12 Oct 2020.

27. FAO. 2016. Methods for estimating comparable rates of food insecurity experienced by adults throughout the world. Rome, FAO. http://www.fao. org/3/i4830e/i4830e.pdf Accessed 12 Oct 2020.

28. Wolfson JA, Ramsing R, Richardson CR, Palmer A. Barriers to healthy food access: Associations with household income and cooking behavior. Prev Med Rep. 2019:13:298-305.

29. Brazier JE, Harper R, Jones NM, O'Cathain A, Thomas KJ, Usherwood J. Validating the SF-36 health survey questionnaire: new outcome measure for primary care. BMJ. 1992;305:160-4.

30. Ware JE. SF-36 health survey update. Spine (Phila Pa 1976). 2000;25:3130-9.

31. Ware JE, Sherbourne CD. The MOS 36-item short-form health survey (SF-36). I. Conceptual framework and item selection. Med Care. 1992;30:473-83.

32. Sheikh KA, Yagoub U, Elsatouhy M, AISanosi R, Mohamud SA. Reliability and validity of the Arabic version of the SF-36 health survey questionnaire in population of Khat Chewers - Jazan Region-Kingdom of Saudi Arabia. Appl Res Qual Life. 2015;10:1-13.

33. Aborisade B, Bach C. Assessing the pillars of sustainable food security. Eur Int J Sci Technol. 2014;3:117-25.

34. Sdepanian VL, de Morais MB. Fagundes-Neto $U$ [Celiac disease: evaluation of compliance to gluten-free diet and knowledge of disease in patients registered at the Brazilian Celiac Association (ACA)]. Arq Gastroenterol. 2001;38:232-9.

35. Leffler DA, Dennis M, Edwards George JB, Jamma S, Magge S, Cook EF, et al. A simple validated gluten-free diet adherence survey for adults with celiac disease. Clin Gastroenterol Hepatol. 2009;7(530-536):536.e531-532.

36. Al Nofaie ND, Al Ahmadi JR, Saadah OI. Health related quality of life among Saudi children and adolescents with celiac disease. Saudi J Gastroenterol. 2020;26:26-31.

37. Rajpoot P, Sharma A, Harikrishnan S, Baruah BJ, Ahuja V, Makharia GK. Adherence to gluten-free diet and barriers to adherence in patients with celiac disease. Indian J Gastroenterol. 2015;34:380-6.

38. Mehta P, Pan Z, Riley MD, Liu E. Adherence to a gluten-free diet: assessment by dietician interview and serology. J Pediatr Gastroenterol Nutr. 2018;66:e67-70.

39. Leffler DA, Edwards George JB, Dennis M, Cook EF, Schuppan D, Kelly CP. A prospective comparative study of five measures of gluten-free diet adherence in adults with coeliac disease. Aliment Pharmacol Ther. 2007;26:1227-35.

40. Muhammad H, Reeves S, Jeanes YM. Identifying and improving adherence to the gluten-free diet in people with coeliac disease. Proc Nutr Soc. 2019;78:418-25.

41. Villafuerte-Galvez J, Vanga RR, Dennis M, Hansen J, Leffler DA, Kelly $C P$, et al. Factors governing long-term adherence to a gluten-free diet in adult patients with coeliac disease. Aliment Pharmacol Ther. 2015:42:753-60.

42. Lee AR, Ng DL, Zivin J, Green PH. Economic burden of a gluten-free diet. J Hum Nutr Diet. 2007;20:423-30.

43. Leffler DA, Edwards-George J, Dennis M, Schuppan D, Cook F, Franko DL, et al. Factors that influence adherence to a gluten-free diet in adults with celiac disease. Dig Dis Sci. 2008;53:1573-81.

44. Tabibian S, Daneshzad E, Bellissimo N, Brett NR, Dorosty-Motlagh AR, Azadbakht $\mathrm{L}$. Association between adherence to the dietary approaches to stop hypertension diet with food security and weight status in adult women. Nutr Diet. 2018;75:481-7.

45. Theodoridis X, Grammatikopoulou MG, Gkiouras K, Papadopoulou SE, Agorastou T, Gkika I, et al. Food insecurity and Mediterranean diet adherence among Greek university students. Nutr Metab Cardiovasc Dis. 2018;28:477-85.

46. Fuchs V, Kurppa K, Huhtala H, Mäki M, Kekkonen L, Kaukinen K. Delayed celiac disease diagnosis predisposes to reduced quality of life and incremental use of health care services and medicines: a prospective nationwide study. United European Gastroenterol J. 2018;6:567-75.

47. Alahmari TM, Asiri AJ, Bilali RM, Qahtani AS, Alqarni MA, Almalwi FA, et al. Quality of life and wellbeing of patients with celiac disease in Aseer Region of Saudi Arabia. Int J Med Sci Public Health. 2018;4:170-4.

48. Nachman F, del Campo MP, González A, Corzo L, Vázquez H, Sfoggia C, et al. Long-term deterioration of quality of life in adult patients with celiac disease is associated with treatment noncompliance. Dig Liver Dis. 2010;42:685-91.

49. Trombetti A, Reid KF, Hars M, Herrmann FR, Pasha E, Phillips EM, et al. Age-associated declines in muscle mass, strength, power, and physical performance: impact on fear of falling and quality of life. Osteoporos Int. 2016;27:463-71.

50. Torres KD, Rosa ML, Moscavitch SD. Gender and obesity interaction in quality of life in adults assisted by family doctor program in Niterói. Brazil Cien Saude Colet. 2016;21:1617-24.

51. Kolotkin RL, Andersen JR. A systematic review of reviews: exploring the relationship between obesity, weight loss and health-related quality of life. Clin Obes. 2017;7:273-89.

52. Kamal S, Aldossari KK, Ghoraba D, Abdelhakam SM, Kamal AH, Bedewi $M$, et al. Clinicopathological and immunological characteristics and outcome of concomitant coeliac disease and non-alcoholic fatty liver disease in adults: a large prospective longitudinal study. BMJ Open Gastroenterol. 2018;5:e000150.

53. Alzaben AS, Turner J, Shirton L, Samuel TM, Persad R, Mager D. Assessing nutritional quality and adherence to the gluten-free diet in children and adolescents with celiac disease. Can J Diet Pract Res. 2015:76:56-63.

54. Rodrigo L, Pérez-Martinez I, Lauret-Braña E, Suárez-González A. Descriptive study of the different tools used to evaluate the adherence to a gluten-free diet in celiac disease patients. Nutrients. 2018;10:1777.

55. Norström F, Lindholm L, Sandström O, Nordyke K, Ivarsson A. Delay to celiac disease diagnosis and its implications for health-related quality of life. BMC Gastroenterol. 2011;11:118.

56. Cranney A, Zarkadas M, Graham ID, Butzner JD, Rashid M, Warren R, et al. The Canadian celiac health survey. Dig Dis Sci. 2007;52:1087-95.

\section{Publisher's Note}

Springer Nature remains neutral with regard to jurisdictional claims in published maps and institutional affiliations. 